Article

\title{
Effect of Exclusive Enteral Nutrition and Corticosteroid Induction Therapy on the Gut Microbiota of Pediatric Patients with Inflammatory Bowel Disease
}

\author{
Lara Hart $^{1}$, Yasamin Farbod ${ }^{1}$, Jake C. Szamosi ${ }^{2}$, Mai Yamamoto ${ }^{3}$, Philip Britz-McKibbin ${ }^{3}$, \\ Camilla Halgren ${ }^{1}$, Mary Zachos ${ }^{1}$ and Nikhil Pai ${ }^{1,2, *(D)}$ \\ 1 Department of Pediatrics, Division of Gastroenterology, Hepatology and Nutrition, McMaster Children's \\ Hospital, McMaster University, Hamilton, ON L8N 3Z5, Canada; lara.hart@medportal.ca (L.H.); \\ yasaminfar71@gmail.com (Y.F.); camilla.halgren@medportal.ca (C.H.); zachosm@mcmaster.ca (M.Z.) \\ 2 Farncombe Family Digestive Health Research Institute, Department of Medicine, McMaster University, \\ Hamilton, ON L8N 3Z5, Canada; jc.szamosi@alumni.utoronto.ca \\ 3 Department of Chemistry and Chemical Biology, McMaster University, Hamilton, ON L8N 3Z5, Canada; \\ yamamm@mcmaster.ca (M.Y.); britz@mcmaster.ca (P.B.-M.) \\ * Correspondence: pain@mcmaster.ca
}

Received: 11 May 2020; Accepted: 1 June 2020; Published: 5 June 2020

\begin{abstract}
Introduction: Exclusive enteral nutrition (EEN) and corticosteroids (CS) are effective induction therapies for pediatric Crohn's Disease (CD). CS are also therapy for ulcerative colitis (UC). Host-microbe interactions may be able to explain the effectiveness of these treatments. This is the first prospective study to longitudinally characterize compositional changes in the bacterial community structure of pediatric UC and CD patients receiving EEN or CS induction therapy. Methods: Patients with diagnoses of CD or UC were recruited from McMaster Children's Hospital (Hamilton, Canada). Fecal samples were collected from participants aged 5-18 years old undergoing 8 weeks of induction therapy with EEN or CS. Fecal samples were submitted for $16 \mathrm{~S}$ rRNA sequencing. The Shannon diversity index and the relative abundance of specific bacterial taxa were compared using a linear mixed model. Results: The clustering of microbiota was the highest between patients who achieved remission compared to patients still showing active disease $(p=0.029)$; this effect was independent of the diagnosis or treatment type. All patients showed a significant increase in Shannon diversity over the 8 weeks of treatment. By week 2, a significant difference was seen in Shannon diversity between patients who would go on to achieve remission and those who would not. Conclusion: The gut microbiota of pediatric UC and CD patients was most influenced by patients' success or failure to achieve remission and was largely independent of the choice of treatment or disease type. Significant differences in Shannon diversity indices occurred as early as week 2 between patients who went on to achieve remission and those who continued to have active disease.
\end{abstract}

Keywords: microbiome; exclusive enteral nutrition; pediatric inflammatory bowel disease; induction of remission

\section{Introduction}

Pediatric inflammatory bowel disease (IBD) comprises $25 \%$ of incident cases of IBD [1]. Exclusive enteral nutrition (EEN) and corticosteroids (CS) are both effective treatment options for the induction of remission in pediatric Crohn's disease (CD), with a clinical remission rate of approximately $80 \%$ for both treatments [2,3]. In the management of pediatric IBD, EEN is recommended as a first-line treatment for pediatric CD. EEN leads to significantly higher rates of mucosal healing and avoids 
short- and long-term corticosteroid side effects that disproportionately affect children [4]. EEN is also beneficial for nutritional rehabilitation, catch-up growth, and improved bone density [4-7]. There is a paucity of literature on the role of EEN in ulcerative colitis (UC) [8].

The mechanisms underlying the effectiveness of EEN in pediatric IBD remain unclear. A microbiome-mediated pathogenesis has been postulated and continues to be studied. Two studies have shown that EEN produces profound changes in a microbial community's structure within as little as $24 \mathrm{~h}$. In particular, Bacteroides spp. and Prevotella spp. significantly decreased, with significant increases in Clostridium leptum abundance following EEN therapy $[5,9]$. These changes correlated with improved disease activity scores. Acute colitis in IBD also carries distinct microbial signatures. Patients with active UC have been shown to have lower Bacteroides spp. and Clostridium spp. [10,11]. Conversely, active CD shows increased Enterobacteriaceae spp. and decreased Clostridium spp. [5].

The role of EEN therapy in UC remains unclear. One study showed improvements in the disease activity and markers of systemic inflammation but no head-to-head comparisons against CS have been performed to date [12]. Clinical guidelines do not describe a role for EEN in the treatment of acute UC [13]. Baseline differences in the taxonomy between IBD phenotypes may account for differences in EEN's efficacy between CD and UC. Additionally, few studies have described the effects of CS for the induction of remission in UC or CD on bacterial community structure, or longitudinal changes in the microbiome of patients on CS or EEN therapy for the induction of remission in pediatric IBD [5,14-16]. The purpose of this study was to report the serial clinical, biochemical, and microbiome changes that occurred in children using EEN versus CS for the induction of remission in pediatric IBD.

\section{Materials and Methods}

\subsection{Study Design and Setting}

We conducted a prospective cohort study on patients admitted to the inpatient Pediatric Gastroenterology service at McMaster Children's Hospital, Hamilton, Ontario (Canada), with a diagnosis of IBD (CD, UC) over a 9-month period. Participants were recruited from September 2015-June 2016. The study received approval from the Hamilton Integrated Research Ethics Board (\#15-365).

\subsection{Participants}

Patients that were 5-18 years old with IBD (CD or UC), diagnosed through histopathology from endoscopic tissue biopsies, serum bloodwork (complete blood count, albumin, sedimentation rate, C-reactive protein (CRP)), fecal calprotectin (FC), and small bowel imaging (magnetic resonance enterography) were approached for recruitment. Patients met the inclusion criteria if they were being initiated on EEN therapy or intravenous CS for the induction of remission of CD or UC. The choice of treatment was at the discretion of the on-service, attending pediatric gastroenterologist. Patients were excluded if they were younger than 5 years old, received antibiotic therapy, or did not require hospital admission for the initiation of treatment. Patients were also excluded if they were started on a 5-ASA (aminosalicylic acid) or biologic therapy for the induction of remission. Two trained research assistants assessed eligibility, obtained informed consent, and addressed all communication requests from patients and families. Patients were approached consecutively over the 9-month study period.

\subsection{Blinding}

This was a non-blinded study. The decision to treat with CS or EEN was at the discretion of the on-service, attending pediatric gastroenterologist.

\subsection{Intervention}

All patients received 8 weeks of EEN or CS treatment. EEN therapy was initiated in the hospital and provided via a nasogastric tube. Patients received Peptamen ${ }^{\circledR} 1.5$ at $120 \%$ of their daily caloric 
needs (based on ideal body weight calculations). Patients were discharged home once the full volume of EEN feeds was reached and tolerated (48-72 $\mathrm{h}$ after initiation). Feeds were condensed to $12-14 \mathrm{~h}$ over the first week. Volumes could be increased if the patient was hungry and the rate could be decreased if there were side effects, such as discomfort or distension. Other than formula, patients were allowed to consume only clear fluids orally. An unrestricted diet was reintroduced after 8 weeks in accordance with our hospital-specific EEN realimentation weaning protocol. Patients on CS therapy were started on treatment in the hospital with IV methylprednisolone at $1 \mathrm{mg} / \mathrm{kg} / \mathrm{day}$ (max: $40 \mathrm{mg} / \mathrm{day}$ ). Once symptoms improved, patients were transitioned to oral CS and discharged home on $1 \mathrm{mg} / \mathrm{kg} / \mathrm{day}$ PO (per os) x 2 weeks, followed by a progressive wean by $5 \mathrm{mg}$ per week. Additional maintenance therapies were initiated as early as two weeks into either EEN or CS induction therapy by the treating pediatric gastroenterologist.

\subsection{Sample Collection}

Fecal samples were collected for microbial analysis before initiating induction therapy and sequentially during induction therapy (days: 0 (pre-treatment), 3, 7; weeks: 2, 4, 6, 8, 12). The samples were immediately stored in a $4{ }^{\circ} \mathrm{C}$ refrigerator and samples brought from home were placed on ice until they could be re-refrigerated in the gastroenterology clinic. Fecal samples were then aliquoted for storage at $-80{ }^{\circ} \mathrm{C}$ within $4-8 \mathrm{~h}$ of collection before sequencing. Serum bloodwork (complete blood count, albumin, CRP) and FC were collected at similar time points to the fecal microbiome sample collection times (Figure 1). Detailed instructions were provided to families and study team members to ensure fecal samples were handled and processed similarly, including between inpatient and outpatient collections.

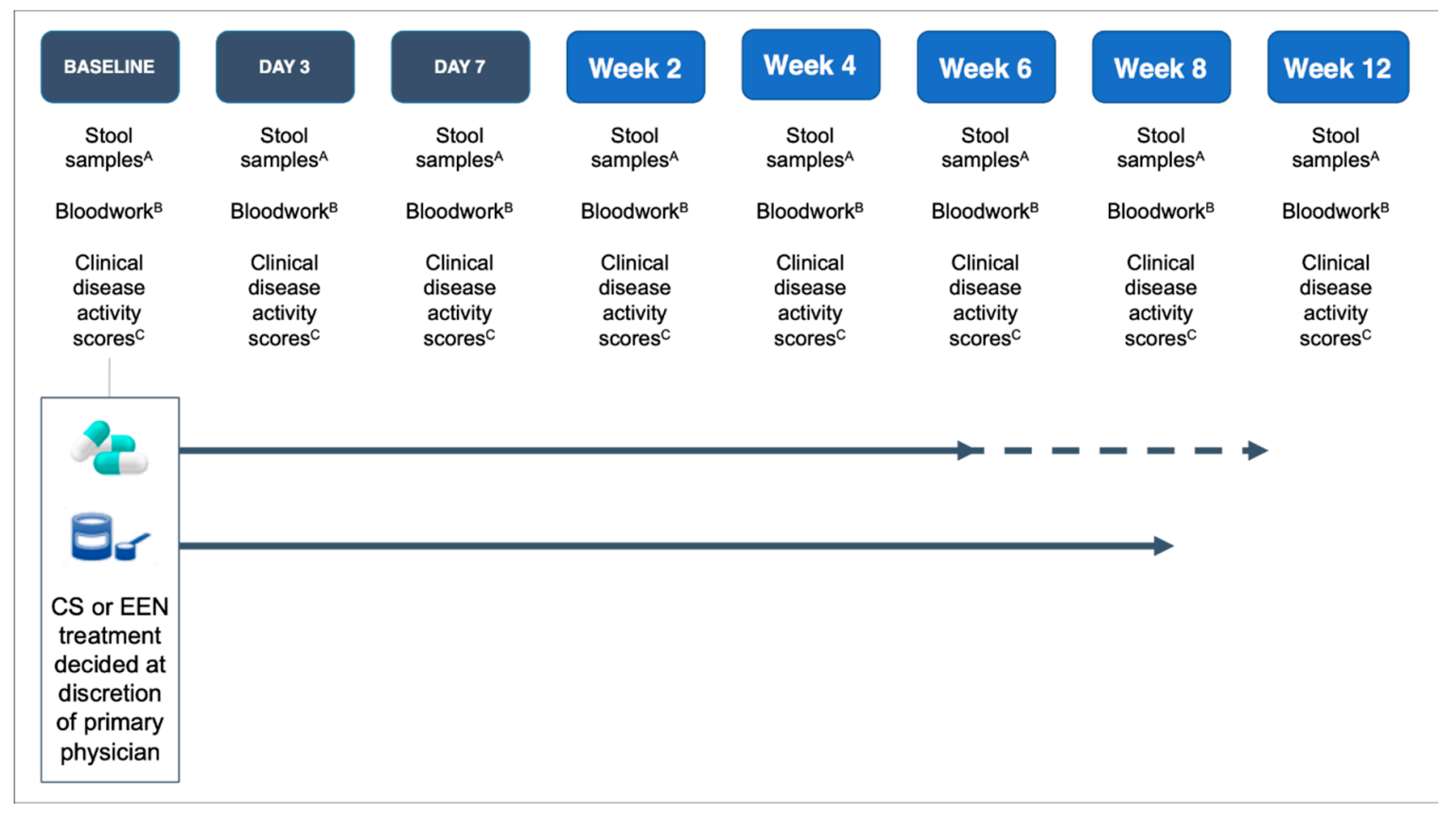

Figure 1. Overview of the study design. CS—corticosteroids, EEN-exclusive enteral nutrition. Note: CS therapy continued for 6-9 weeks at weaning doses; EEN therapy continued for 8 weeks. A Stool samples: fecal calprotectin and fecal microbiota. ${ }^{B}$ Bloodwork: complete blood count (CBC), albumin, and C-reactive protectin (CRP). ${ }^{C}$ Clinical disease activity scores: Pediatric Ulcerative Colitis Activity Index (PUCAI) and Pediatric Crohn's Disease Activity Index (PCDAI). 


\subsection{Clinical Assessment}

Once weekly, while on induction therapy, patients received a telephone call from trained research assistants to assess clinical symptoms. Clinical assessments were measured using the Pediatric UC Activity Index (PUCAI) or a modified Pediatric CD Activity Index (PCDAI) (excluding the physical exam information) $[17,18]$. Clinical improvement was defined as a decrease in the PUCAI or modified PCDAI from baseline enrolment, and clinical remission was defined as a score of $\leq 10$ for each scale. The response to treatment was classified in terms of active disease, clinical response, clinical remission, or full remission. Active disease was defined as all baseline clinical and biochemical (CRP, albumin, hemoglobin, FC) parameters. Clinical response was defined as improvement in PCDAI/PUCAI and biochemical parameters from active disease. Clinical remission was defined as clinical response and improvement in biochemical markers from active disease. Full remission was defined as clinical remission and biochemical markers within normal limits $(\mathrm{FC}<250 \mathrm{mcg} / \mathrm{g})$.

\subsection{Microbial Community Structure Analysis}

Microbial community profiling was conducted by extracting genomic DNA from patient and donor stool samples using a protocol described previously, which enhances total DNA recovery [19]. After genomic DNA extraction, the V3 region of the 16S ribosomal RNA gene was amplified (total polymerase chain reaction volume of $50 \mu \mathrm{L}$ ( 25 pmol of each primer, $200 \mu \mathrm{L}$ each deoxynucleoside triphosphate, $1.5 \mathrm{mM} \mathrm{MgCl}_{2}$, and Taq polymerase), divided into triplicate for greater efficiency). Samples were sequenced using the Illumina MiSeq platform (San Diego, CA, USA), as per manufacturer's instructions and raw sequence reads were processed as described previously [20]. The stool microbiota was sampled to a mean depth of 103,341 reads per sample (median $=87,509$ ).

\subsection{Statistical Analysis}

For clinical outcomes, statistical differences between pairwise comparisons were calculated using the Mann-Whitney U test, with significance defined as $p<0.05$. Microbiome data were analyzed in R using the Phyloseq package (version 3.11; Boston, MA, USA). Samples were ordinated using a principal coordinate analysis (PCoA) of Bray-Curtis distances to visualize clustering. The differential abundance of bacterial genera was tested using the generalized linear model implemented in DESeq2 (version 3.11; Boston, MA, USA) and restricted to those genera that were present in at least $50 \%$ of the samples. We omitted any mean $<10$ reads/100,000 (relab $<0.001)$, max $<100$ reads/100,000 (relab < 0.1). If $p(0) \leq 0.7$, we performed DESeq directly, and if $p(0)>0.7$, we binarized and performed logistic regression on the results. Spearman correlations of the genus relative abundance with hemoglobin, albumin, CRP, and FC were also restricted to those genera present in at least $50 \%$ of the samples using pairwise-complete observations.

\section{Results}

\subsection{Participants}

Thirty patients were enrolled (69\% male); 16 patients received EEN and 14 received CS therapy. Of these, all patients receiving EEN had CD and $29 \%$ of patients receiving CS had CD. A total of $63 \%$ of patients were newly diagnosed and undergoing their first induction therapy. Baseline bloodwork and FC values were similar in patients who received EEN and CS (Table 1). 
Table 1. Baseline characteristics of patients starting induction therapy with EEN or CS.

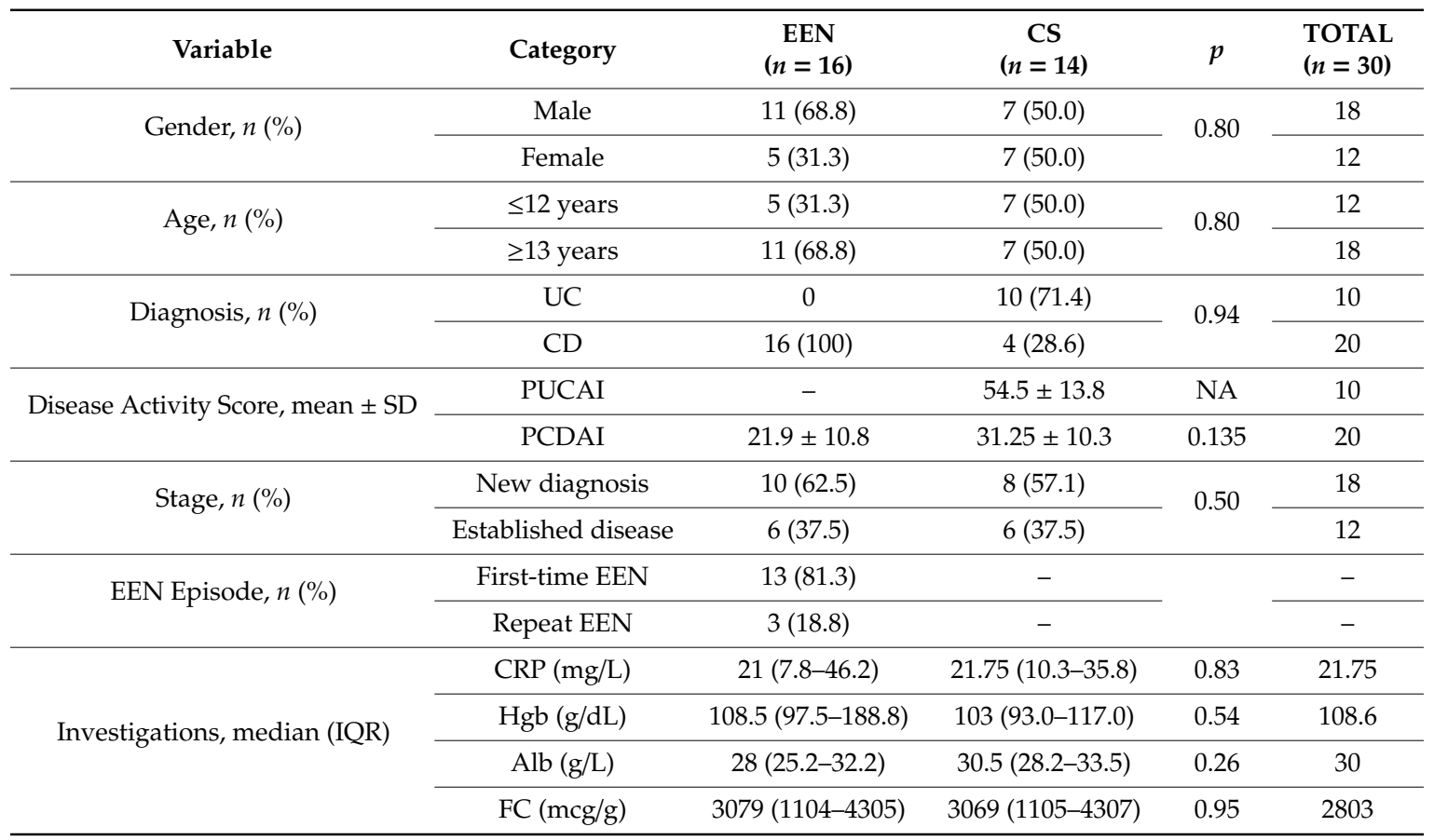

EEN-exclusive enteral nutrition; CS-corticosteroids; Alb-albumin; CRP-C-reactive protein; FC-fecal calprotectin; Hgb-hemoglobin; PCDAI-Pediatric Crohn's Disease Activity Index; PUCAI-Pediatric Ulcerative Colitis Activity Index.

\subsection{Response to EEN or CS}

A total of $60 \%(n=6)$ of patients with UC and $90 \%(n=18)$ of patients with CD achieved clinical remission by the end of therapy at week 8 . Of the 6 patients who did not achieve remission, $83 \%(n=5 ; 1 \mathrm{CD}, 4 \mathrm{UC})$ received $\mathrm{CS}$ and $17 \%(n=1 ; \mathrm{CD})$ received EEN. One patient required a second hospitalization for disease relapse (despite CS therapy). For both the EEN and CS treatment groups, there was a significant decrease in CRP (EEN, CS: $p=0.03$ ), increase in albumin (EEN, CS: $p=0.02$ ), and decrease in FC (EEN: $p=0.033$, CS: $p<0.005)$ after therapy (Table 2).

Table 2. End of treatment characteristics of patients receiving EEN or CS.

\begin{tabular}{ccccc}
\hline Variable & Category & $\begin{array}{c}\text { EEN } \\
(n=16)\end{array}$ & $\begin{array}{c}\text { CS } \\
(n=14)\end{array}$ & $p$ \\
\hline \multirow{2}{*}{ Disease Activity Score, mean $\pm \mathrm{SD}$} & PUCAI & - & $17.5 \pm 27.5$ & - \\
\cline { 2 - 5 } & PCDAI & $2.5 \pm 3.8$ & $8.3 \pm 10.4$ & 0.18 \\
\hline \multirow{2}{*}{ Investigations, median (IQR) } & $\mathrm{CRP}(\mathrm{mg} / \mathrm{L})$ & $1.45(0.3-14.8)$ & $0.7(0-5.7)$ & 0.67 \\
\cline { 2 - 5 } & $\mathrm{Hgb}(\mathrm{g} / \mathrm{dL})$ & $119.5(115.8-130.0)$ & $113(100.3-128.0)$ & 0.39 \\
\cline { 2 - 6 } & $\mathrm{Alb}(\mathrm{g} / \mathrm{L})$ & $36(31.5-39.0)$ & $35.5(33.5-40.5)$ & 0.78 \\
\cline { 2 - 6 } & $\mathrm{FC}(\mathrm{mcg} / \mathrm{g})$ & $274(104.7-1010.0)$ & $546(110.6-1093.0)$ & 0.91 \\
\hline
\end{tabular}

EEN-exclusive enteral nutrition; CS-corticosteroids; Alb-albumin; CRP-C-reactive protein; FC—fecal calprotectin; Hgb—hemoglobin; PCDAI—Pediatric Crohn's Disease Activity Index; PUCAI—Pediatric Ulcerative Colitis Activity Index.

\subsection{Microbiota Composition in EEN vs. CS Treated Patients}

When all time points were included, the microbiota of patients who had CD or UC clustered differently based on principal coordinate analysis (Figure 2) and a PERMANOVA test $\left(\mathrm{R}^{2}=0.047\right.$, $p=0.001$ ). Bacterial taxon abundances were measured over the course of treatment between patients 
who received CS ( $n=14 ; 10 \mathrm{UC}, 4 \mathrm{CD})$ and EEN therapy ( $n=16 ; 0 \mathrm{UC}, 16 \mathrm{CD})$. Overall, as patients progressed from active disease to full remission, greater abundances of the following genera were found in both treatment groups: Blautia $(p=0.045)$, Sellimonas $(p=0.002)$, and uncharacterized bacteria from family Ruminococcaceae ( $p=0.0032)$ (Figure 3A). The abundances of three genera were reduced as the patients' health improved: Granulicatella $(p=0.027)$, Haemophilus $(p=0.0001)$, and Streptococcus $(p=0.002)$ (Figure 3B). In two taxa, there was a significant association of the disease state with abundance but no clear linear pattern of change: Bacteroides $(p=0.03)$ and uncharacterized bacteria from family Peptostreptococcaceae $(p=0.00005)$ (Figure 3C). As patients progressed from active disease to full remission, while not meeting statistical significance, greater abundances were also found in both treatment groups for Faecalibacterium and Fusobacterium that were absent from more than $50 \%$ of the samples in the remission state. These changes were seen regardless of treatment type at the genus and phylum levels (Figure 3D,E).

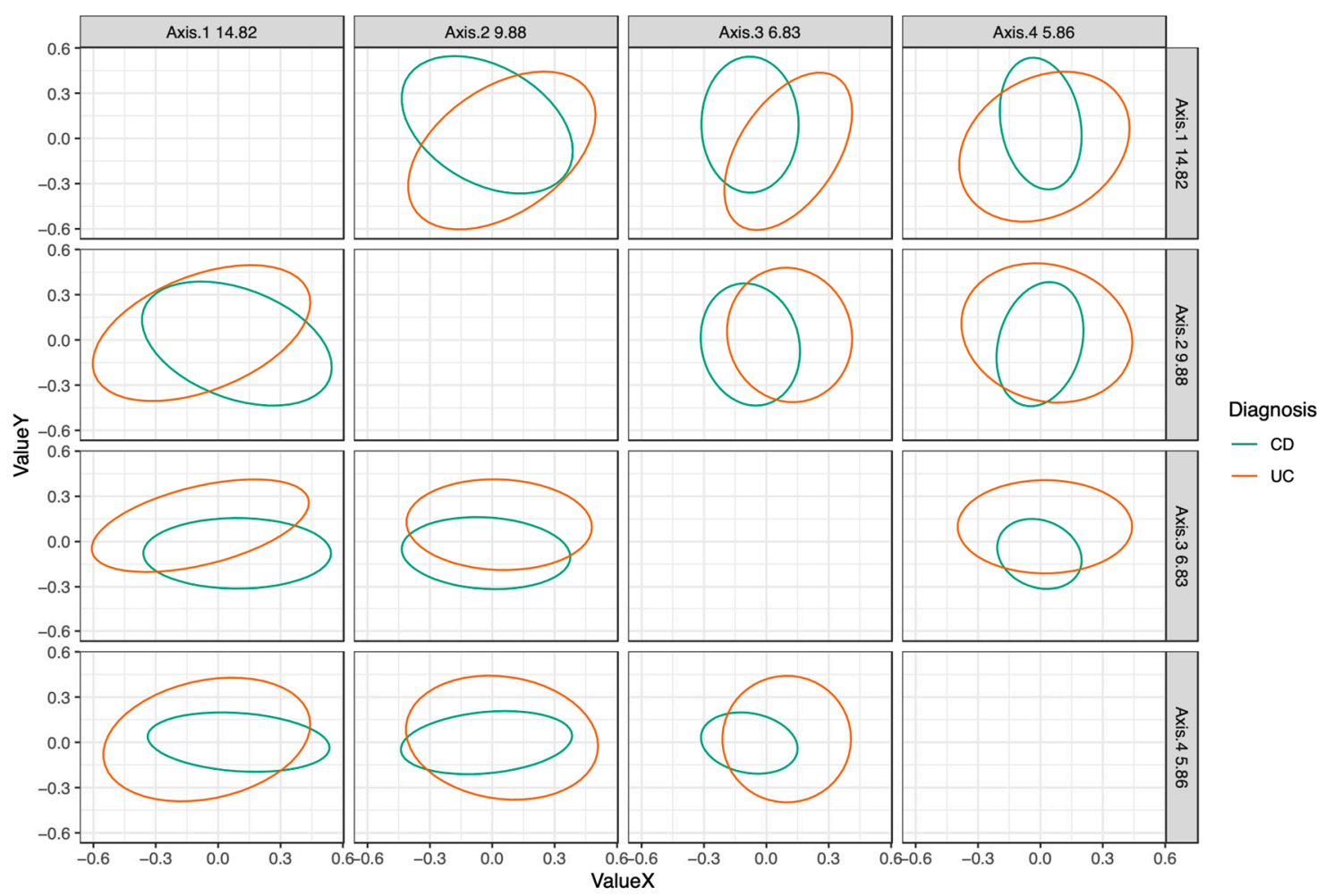

Figure 2. Principal coordinate analysis of $C D$ and UC fecal microbiota at baseline (active disease). Fecal microbiota collected from 20 patients with CD, 10 patients with UC with active disease before the onset of treatment. Bacterial taxa clustered differently between diagnoses. CD-Crohn's disease, UC-ulcerative colitis. 


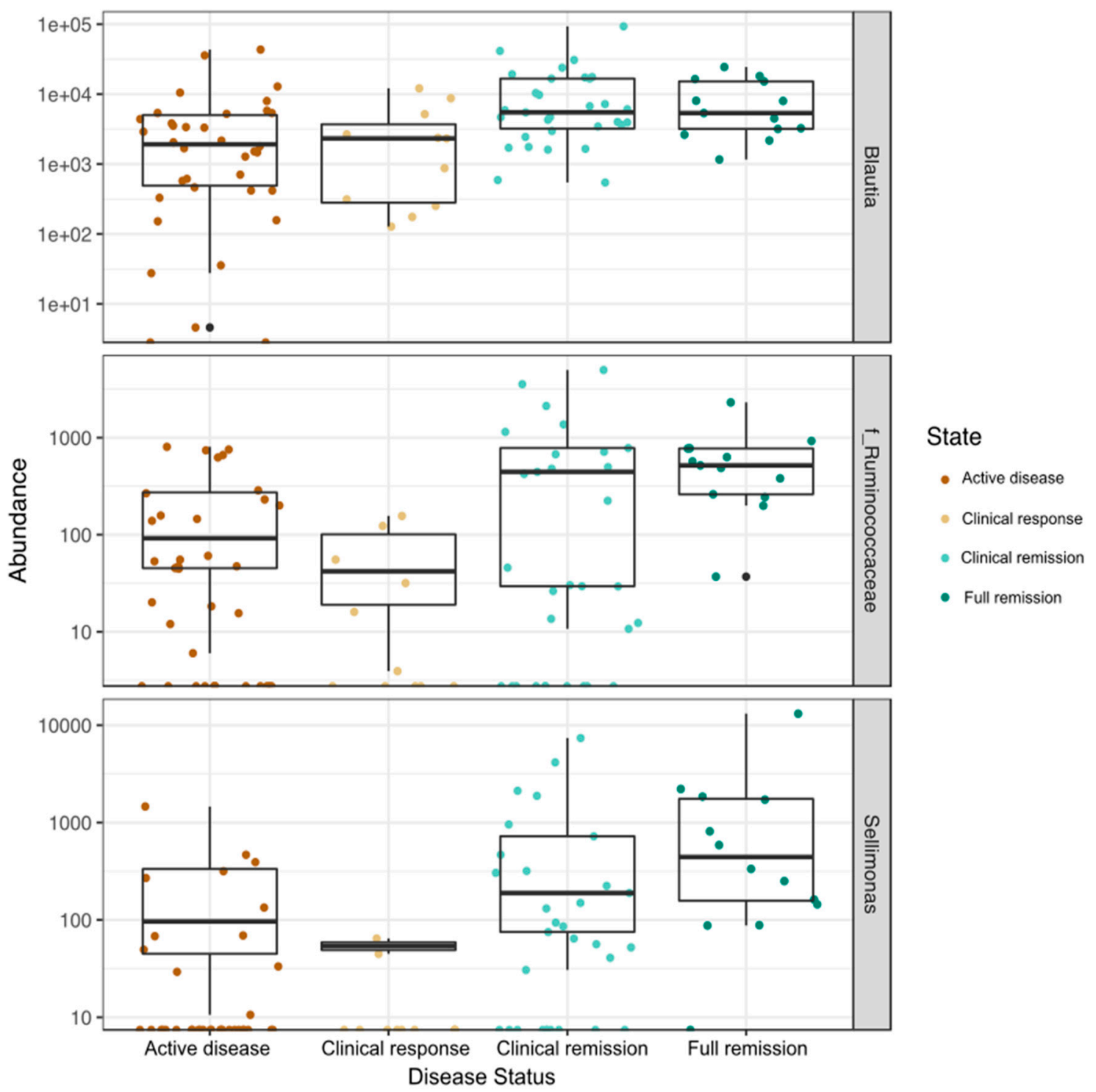

(A)

Figure 3. Cont. 


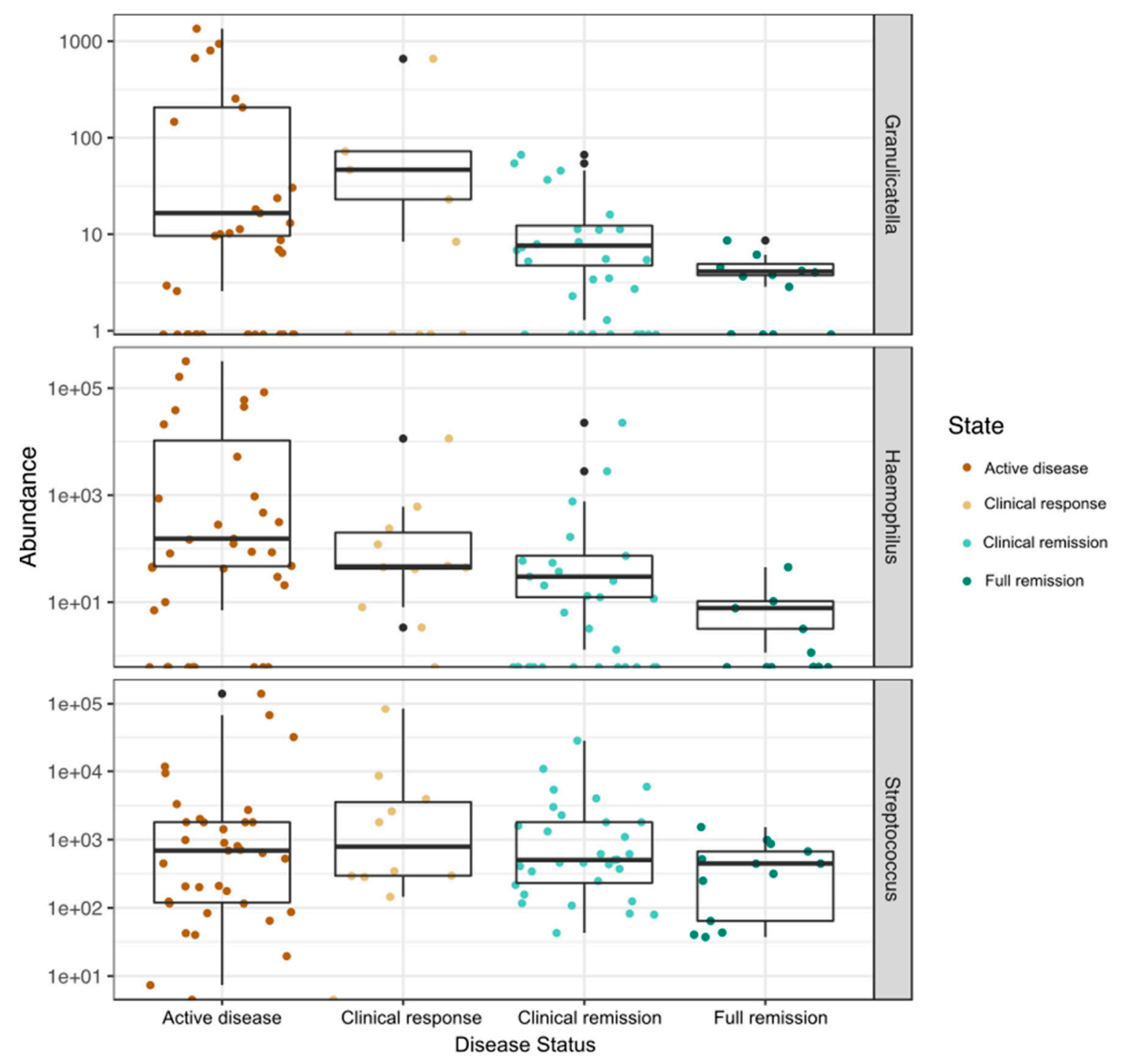

(B)

Figure 3. Cont. 


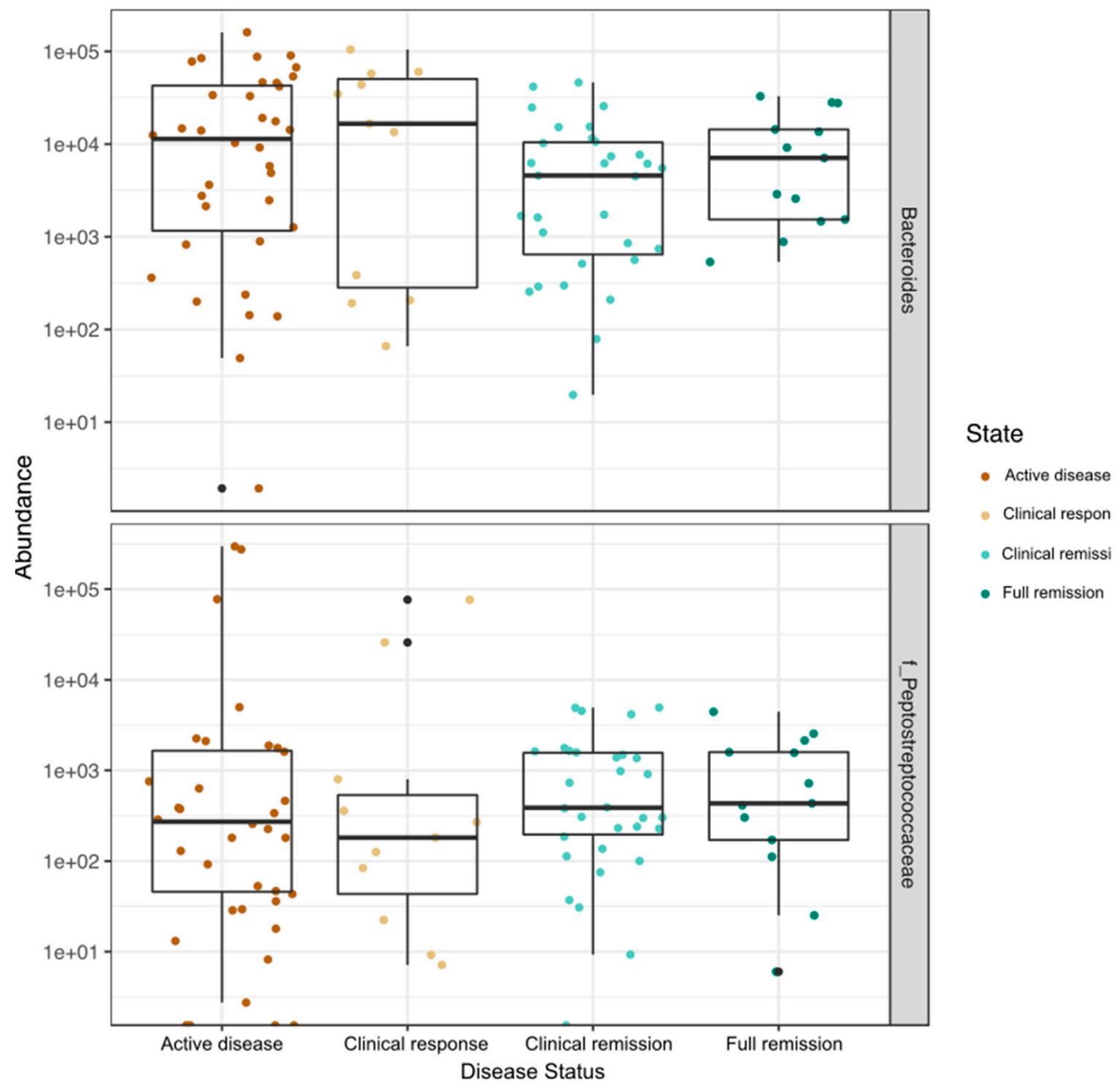

(C)

Figure 3. Cont. 

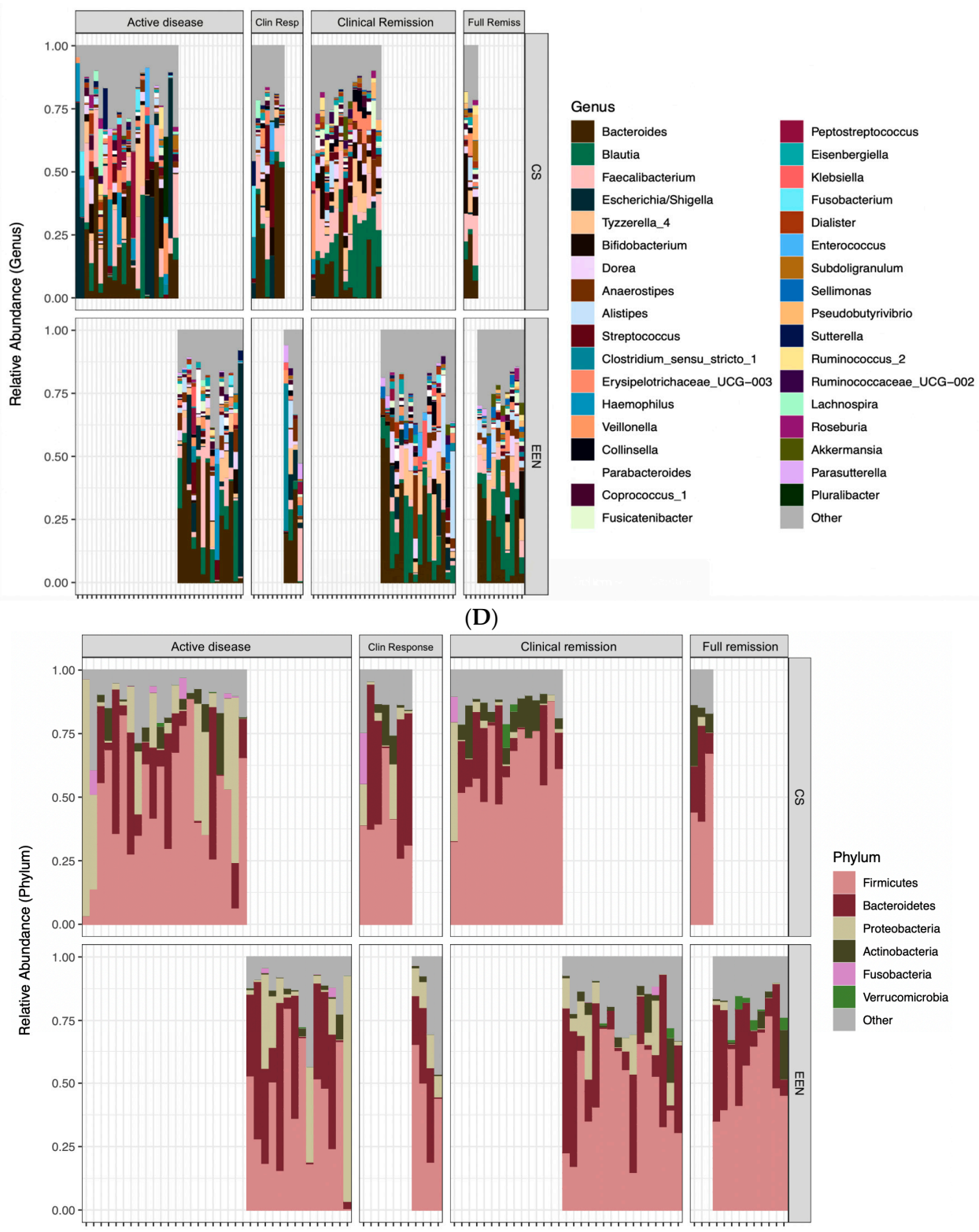

(E)

Figure 3. (A) Taxa that increased in abundance with disease response. Taxa that increased abundance over the course of treatment (as symptoms improved) were Blautia $(p=0.045)$, Sellimonas $(p=0.002)$, and uncharacterized bacteria from family Ruminococcaceae $(p=0.0032)$. (B) Taxa that decreased in abundance with disease response. Taxa that decreased in abundance over the course of treatment (as symptoms improved) were Granulicatella $(p=0.027)$, Haemophilus $(p=0.0001)$, and Streptococcus $(p=0.002)$. (C) Taxa with significant changes in abundance but an unclear disease response. There was a significant association of disease state with abundance but no clear linear pattern of change with Bacteroides ( $p=0.03$ ) and uncharacterized bacteria from family Peptostreptococcaceae $(p=0.00005)$. (D) The relative abundance of bacterial genera in terms of the treatment and disease state. The microbial community structure at the genus level of CD and UC patients receiving either CS or EEN therapy in terms of the phase of treatment. Greater abundances of Bacteroides, Blautia, and Faecalibacterium were noted from an active disease at the clinical response, clinical remission, and full remission stages in both treatment groups. (E) The relative abundance of bacterial phylum in terms of the treatment and disease state. The microbial community structure at the phylum level of $\mathrm{CD}$ and UC patients receiving either CS or EEN therapy in terms of the phase of treatment. CS-corticosteroids, EEN-exclusive enteral nutrition. 
At the end of therapy (week 8), although not reaching statistical significance, patients treated with EEN ( $n=16 ; 16 \mathrm{CD}$ ) showed depletion in bacteria from the Fusobacterium, Escherichia/Shigella, and Veillonella genera. In patients treated with CS $(n=14 ; 10 \mathrm{UC}, 4 \mathrm{CD})$, reductions in Alistipes, Veillonella, and Fusobacterium genera were observed. These changes were independent of the patients' underlying disease type (CD, UC) (Figure 4).
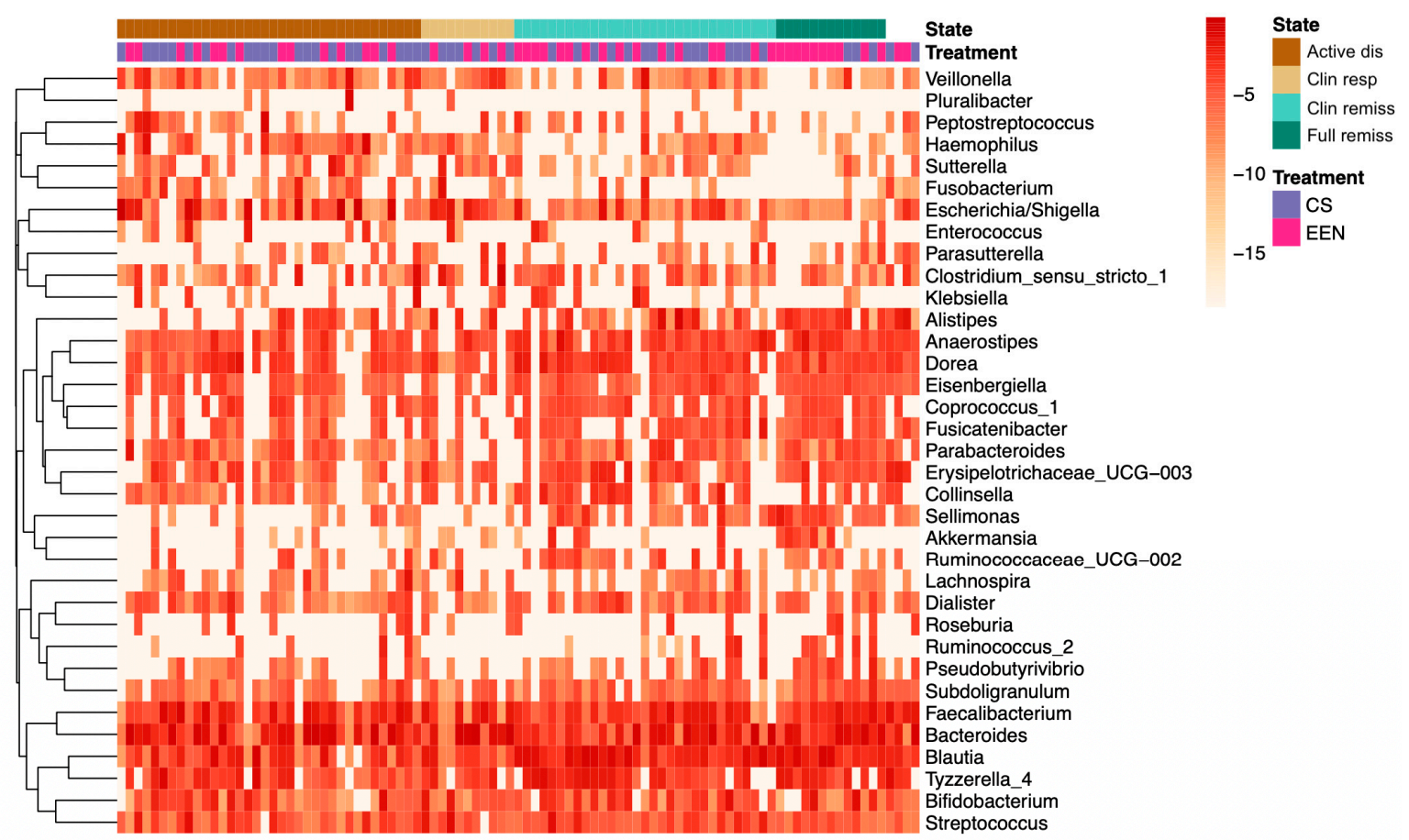

Figure 4. Heatmap illustrating the change in the taxonomic composition of the fecal microbiome in terms of the disease state and treatment type. Changes in the relative abundance of genera across the treatment state. At the clinical response stage, patients treated with EEN showed depletion in Fusobacterium, Escherichia/Shigella, and Veillonella, compared to patients treated with CS, who showed reductions in Alistipes, Veillonella, and Fusobacterium genera. CS-corticosteroids, EEN-exclusive enteral nutrition.

The change in microbiota composition was also plotted based on clinical and serological markers of response to treatment (active disease, clinical response, clinical remission, full remission). A permutation test of the microbial community dispersions during active disease between UC and CD showed significant differences between the two diagnoses during active disease $(\mathrm{F}(3)=7.1094, p=0.011)$. Tighter clustering was observed in patients after 8 weeks of therapy, independent of treatment type, while the greatest dispersion was seen in patients during active disease before treatment was initiated (Figure 5A,B).

We also noted a significant increase in Shannon diversity over time $(\mathrm{F}(1)=8.019, p=0.006)$ and across the disease states $(\mathrm{F}(3)=4.389, p=0.007)$. This effect did not vary based on the type of treatment received (Figure 6A,B). We also found a significant difference in Shannon diversity at week 2 of treatment between patients who would go on to achieve remission at end of therapy (week 8) and those who would not. This was found in all patients, regardless of whether they received CS or EEN $(\mathrm{F}(1)=5.074, p=0.044)$ (Figure $6 \mathrm{C})$, and was not present during the active disease state at baseline (week 0). 


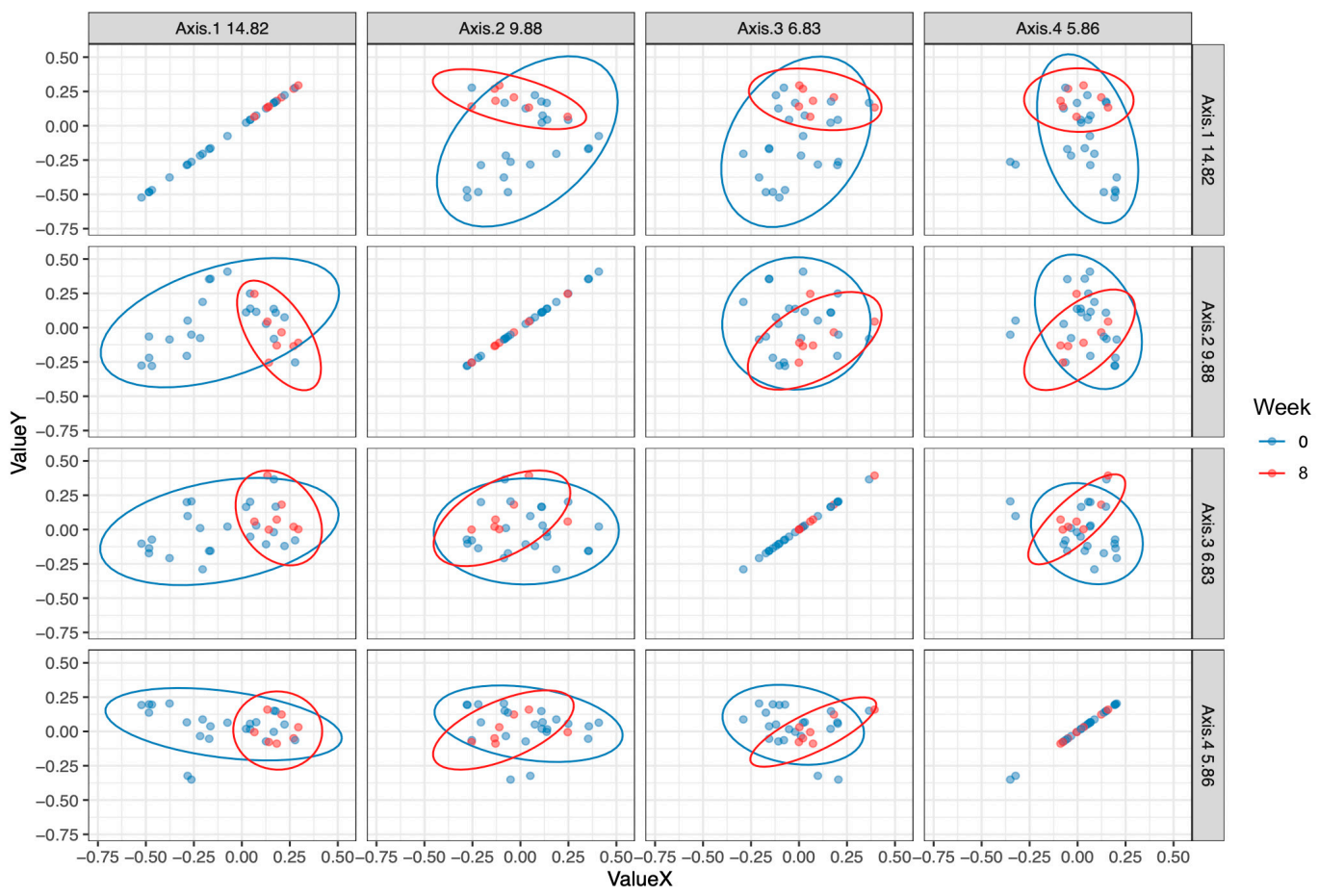

(A)

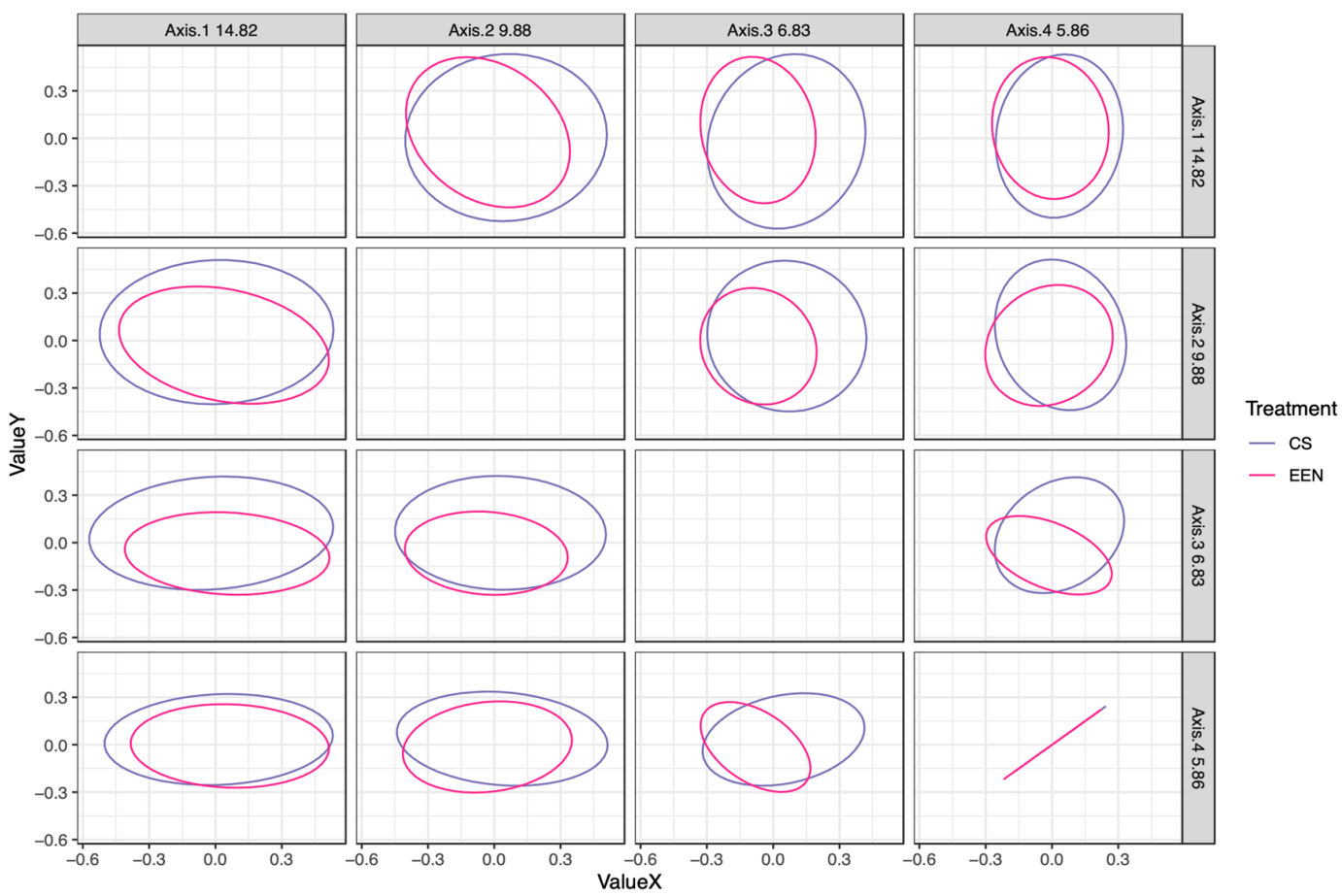

(B)

Figure 5. (A) Principal coordinate analysis of fecal microbiota at baseline (active disease) and end of treatment. Greater clustering was observed at the end of treatment independent of the disease type or treatment. (B) Principal coordinate analysis of the fecal microbiota in terms of the treatment type at the end of treatment. Principal coordinate analysis of patients at the end of treatment (week 8) in terms of the CS or EEN treatment type. Clustering was observed between all samples, independent of the treatment type. CS—corticosteroids, EEN-exclusive enteral nutrition. 

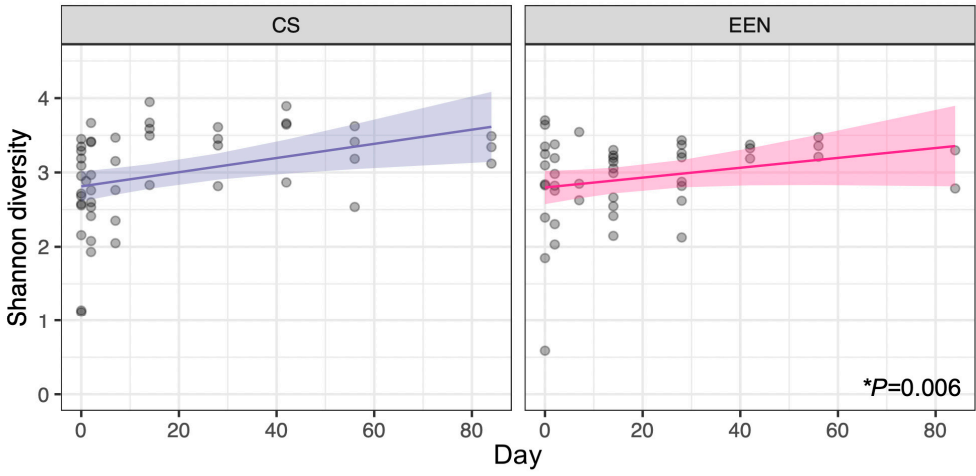

\section{Treatment}

- cs

- EEN

(A)

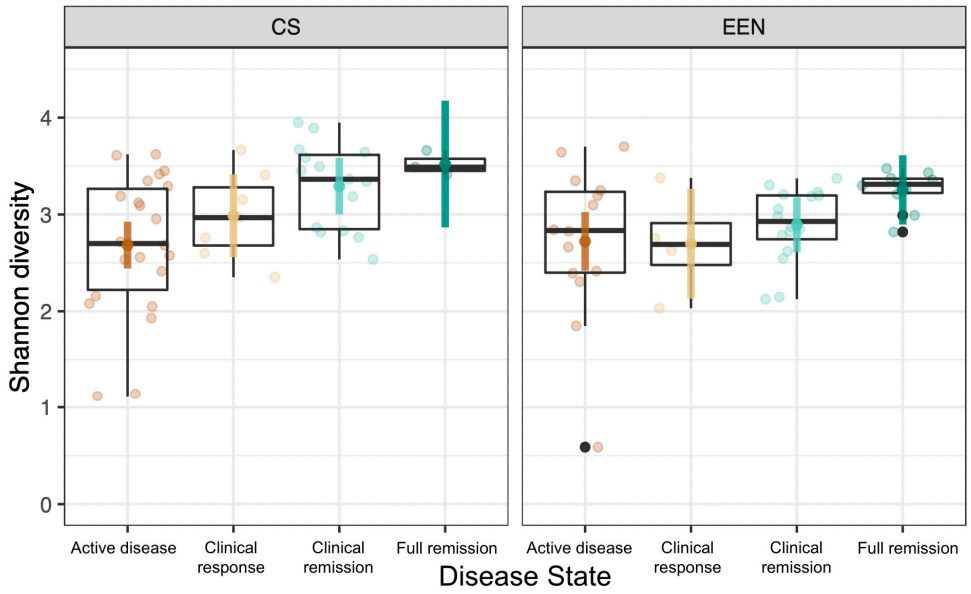

State

Active disease

Clinical response

1. Clinical remiss

| Full remission

(B)

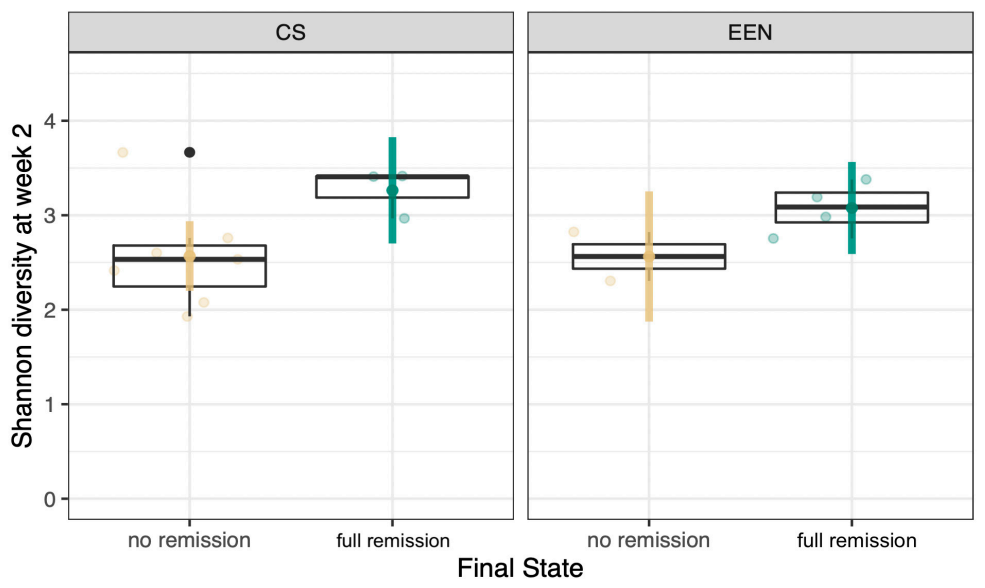

Final State

no remission

full remission

(C)

Figure 6. (A) Shannon diversity over time separated by treatment. A significant increase in Shannon diversity was observed over time (day 0 to day 84$)$, independent of the treatment received $(p=0.006)$. (B) Shannon diversity over the disease state separated by treatment. A significant increase in Shannon diversity was observed over the disease state (active disease, clinical response, clinical remission, full remission) in both CS- and EEN-treated patients ( $p=0.007)$. (C) Shannon diversity at week 2 compared to the final remission status at the end of treatment, separated by treatment type. A significant difference in Shannon diversity at week 2 was observed between patients who achieved remission at the end of treatment and those who did not achieve remission $(p=0.044)$. CS-corticosteroids, EEN-exclusive enteral nutrition. 


\subsection{Correlations between Bacterial Genera and Clinical, Fecal, and Serum Markers of Disease Status}

The genera Sellimonas, Ruminiclostridium, and families Ruminococcaceae and Erysipelotrichaceae were strongly positively correlated with serum hemoglobin and albumin levels, while Veilonella, Escherichia/Shigella, and Haemophilus were strongly negatively correlated with both serum levels. CRP and FC were negatively correlated with Sellimonas, Ruminiclostridium, and families Ruminococcaceae and Erysipelotrichaceae (Figure 7).

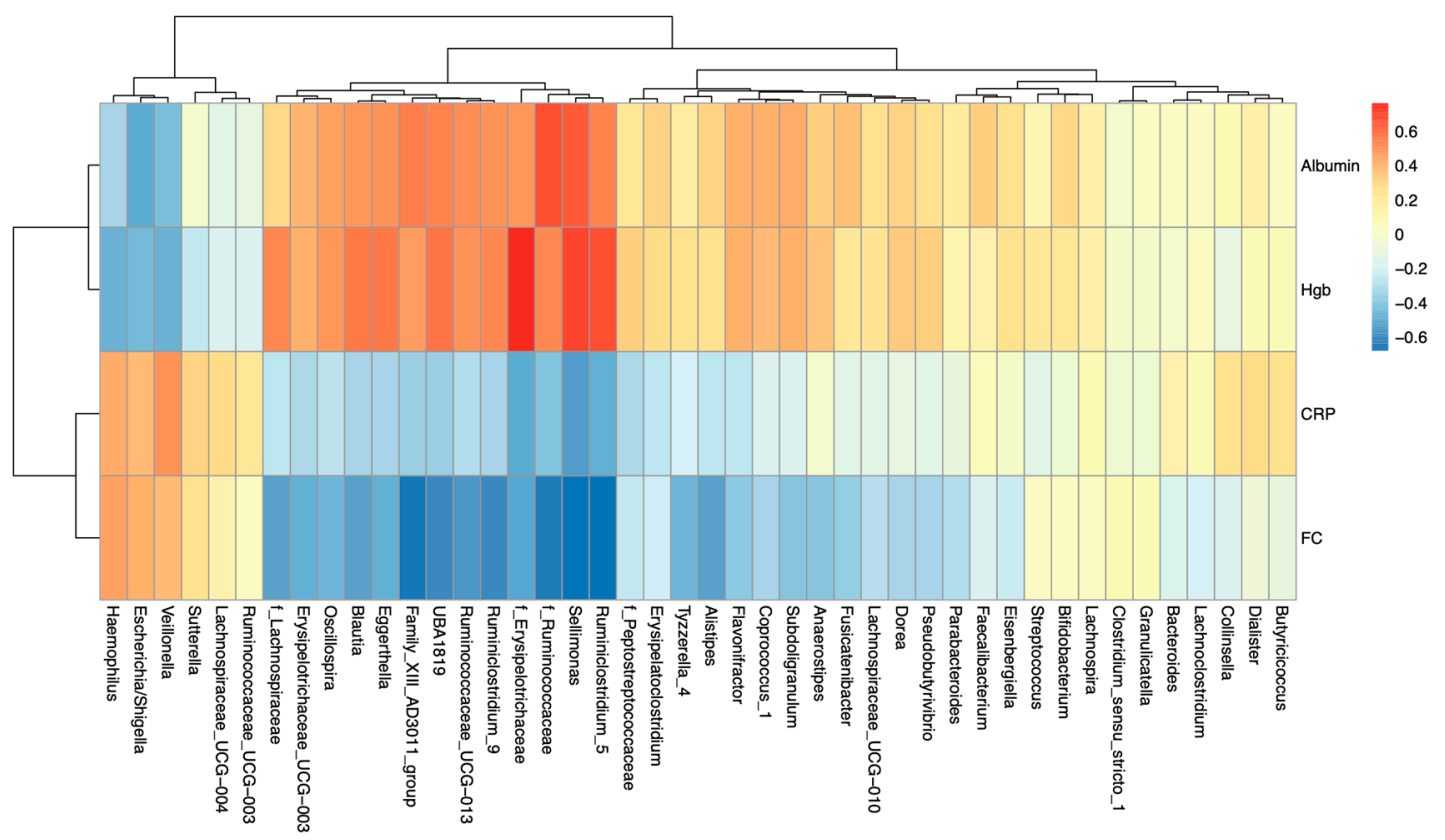

Figure 7. Correlation of fecal and serum markers of disease with bacterial genera. Genera Sellimonas and Ruminiclostridium positively correlated with serum $\mathrm{Hgb}$ and albumin levels, and negatively correlated with CRP and FC. Genera Veilonella and Escherichia/Shigella negatively correlated with serum Hgb and albumin levels. Hgb-hemoglobin, CRP-C-reactive protein, FC—fecal calprotectin

\section{Discussion}

This prospective cohort study in pediatric UC and CD was designed to assess dynamic changes in the intestinal microbiota profiles of patients receiving eight weeks of induction therapy using CS or EEN. Our study found that regardless of disease phenotype or therapy modality, at the end of 8 weeks, as patients' health improved, their microbiome communities were significantly more closely clustered than they were before treatment began.

Both treatment options achieved the induction of remission in the majority of UC and CD patients. Following treatment with EEN, the median decreases in CRP and FC were $19.6 \mathrm{mg} / \mathrm{L}$ and $2805 \mathrm{mcg} / \mathrm{g}$, respectively; PCDAI scores decreased by 19.0 points. Patients receiving treatment with CS had a median decreases in CRP and FC of $21.1 \mathrm{mg} / \mathrm{L}$ and $2523 \mathrm{mcg} / \mathrm{g}$, respectively; there was a decrease in PCDAI of 23.0 points in CD patients and a decrease in PUCAI of 37.0 points in UC patients. These results were consistent with previous work demonstrating a response to CS and EEN treatments in UC and CD $[2,4,8]$.

During the treatment phase in our study, a significant shift was demonstrated in the abundances of some microbial taxa in patients from both UC and CD groups. Patients who were treated with EEN showed a marked depletion in bacteria from the Fusobacterium, Escherichia/Shigella, and Veillonella genera. This is consistent with previous literature suggesting that the treatment response in CD is associated with changes in these taxa [14]. In patients treated with CS ( $n=14 ; 10 \mathrm{UC}, 4 \mathrm{CD})$, reductions in the Alistipes, Veillonella, and Fusobacterium genera were observed. Due to sample size limitations, we did not independently assess taxonomic differences in CS patients with UC and CD separately. 
Further, patients who achieved a state of remission had an increased abundance of Blautia, Sellimonas, and the family Ruminococcaceae, as well as a decrease in the abundance of Granulicatella, Haemophilus, and Streptococcus genera. These results suggest that if therapy is successful in inducing remission, the microbial community shifts toward a similar healthy end-state (remission microbiota profile), regardless of the patient's underlying disease type or induction therapy. This could also suggest that at the mucosal level, UC and CD lie along a continuum of a common disruptive host-microbe pathway rather than showing two fundamentally distinct microbial disease states. This has previously been described by other investigators through genome-wide association studies, suggesting that shared genes coding for immune loci are found across both CD and UC patients [21-23].

We found that as patients entered remission, they showed a significant increase in Shannon diversity compared to during the active disease state. This occurred in patients treated with CS or EEN, which has been supported by both Schwerd et al. [24] and D'Argenio et al. [25]. This has been challenged by other investigators, where alpha-diversity was actually found to decrease in patients treated with EEN. We propose that our conclusions must be evaluated in consideration of our sample size, having collected 45 stool samples throughout the study among 16 patients who received EEN. Yet, other investigators have also drawn conclusions from similarly small sample sizes. Gerasimidis et al. (20 patients; $n=68$ samples), Leach et al. (6 patients; actual number not reported, $n=48$ samples maximum), and Kaakoush et al. (5 patients; actual number not reported, presumed $n=25-45$ samples) all reported a significant decrease in alpha-diversity over time among pediatric patients receiving EEN $[5,9,26]$. All three groups reported on similarly limited sample sizes and stool samples for analysis.

Unique to our study, we also noted a significant predictive effect of Shannon diversity as early as week 2 of treatment. Patients who went on to achieve remission by the end of therapy (week 8) had a significantly higher Shannon diversity in both treatment groups (CS, EEN) at week 2 compared to those who did not achieve remission. If reproducible, this finding would have significant implications for predicting responders from non-responders early in the treatment course. This would have appreciable impacts on the quality of life given that at week 2, patients generally have between 6-10 more weeks of treatment remaining. It could also provide a further biomarker of response to therapy, in conjunction with clinical findings and bloodwork investigations.

Several bacterial taxa were associated with changes in clinical, serum, and fecal markers of disease status. Ruminococcus was positively associated with improvements in hemoglobin levels. This was consistent with other investigators who have shown similar correlations between Ruminococcus and improvement in hemoglobin in recipients of EEN [14]. For the first time, we report that reductions in CRP and FC were inversely correlated with changes in Ruminococcaceae.

There is little data available on the role of EEN in UC treatment. Animal models of dextran sulfate sodium (DSS)-induced colitis have shown that EEN can reduce cytokine expression and improve tissue histology [27]. A small case series from Wedrychowicz et al. studied the results of 39 pediatric UC and CD patients receiving EEN for induction therapy. This study did not compare results against corticosteroid induction but improvements in CRP, Hgb, clinical disease activity, and weight-for-age $z$ scores were noted [12].

Our study has several limitations, particularly the small number of patients included and samples obtained, and therefore there was a treatment heterogeneity across CD and UC diagnoses. Our numbers reflect the difficulties in performing such trials in children and adolescents with IBD, where validated guidelines promoting the use of CS as first-line therapy in UC exist [8]. In our study, no UC patients received EEN as induction therapy. While our study protocol allowed primary pediatric gastroenterologists to assign their choice of induction treatment without restriction, patient and physician discomfort of assigning an atypical first-line therapy for acute UC limited our ability to compare the clinical and microbiota changes of this treatment approach. This precluded our ability to assess the role of EEN in patients with UC. Further, our institution heavily supports and encourages the use of EEN as first-line therapy in CD patients in accordance with international guidelines on the treatment of pediatric CD [2]. These limited the number of patients in the CD group who received CS 
for induction therapy but do provide some preliminary data that may be followed up on in future studies of a larger sample size.

\section{Conclusions}

In summary, our results suggest that $\mathrm{UC}$ and $\mathrm{CD}$ patients developed remission microbiota profiles that were similar. This trend was independent of the induction treatment choice (EEN vs. CS) and baseline diagnosis. While EEN therapy continues to be an important treatment option for $C D$, and CS remains the first-line treatment for patients with UC, broad changes in microbiota appear to be driven by the success or failure to achieve clinical remission more than treatment choice or the diagnosis itself. Further, a higher Shannon diversity at week 2 may predict the likelihood of remission at the end of therapy. Current work is underway to characterize dynamic changes in the fecal and urine metabolome of pediatric IBD patients to better delineate treatment responses to therapy.

Author Contributions: Conceptualization, L.H., M.Z. and N.P.; data curation, L.H., Y.F. and C.H.; formal analysis, J.C.S. and N.P.; funding acquisition, L.H. and N.P.; investigation, Y.F., P.B.-M. and M.Z.; methodology, L.H., M.Y., P.B.-M., J.C.S., M.Z. and N.P.; supervision, N.P.; validation, J.C.S.; writing-original draft, L.H., J.C.S., and N.P.; writing-review and editing, L.H., M.Y., P.B.-M., J.C.S., M.Z. and N.P. All authors have read and agreed to the published version of the manuscript.

Funding: The research was funded by the Canadian Association of Gastroenterology Resident Research Grant and KidsDigHealth Fund (Division of Pediatric Gastroenterology and Nutrition, McMaster Children's Hospital).

Acknowledgments: The authors wish to thank the Division of Pediatric Gastroenterology and Nutrition, and the nurses of Ward 3Y at McMaster Children's Hospital for their introductions to the patient participants and support with sample collection. We also extend our gratitude to Michael Surette and the Farncombe Family Digestive Health Research Institute for his assistance with conducting this work.

Conflicts of Interest: The authors declare no conflict of interest.

\section{References}

1. Sauer, C.G.; Kugathasan, S. Pediatric Inflammatory Bowel Disease: Highlighting Pediatric Differences in IBD. Med. Clin. N. Am. 2010, 94, 35-52. [CrossRef]

2. Ruemmele, F.M.; Veres, G.; Kolho, K.L.; Griffiths, A.; Levine, A.; Escher, J.C.; Dias, A.J.; Barabino, A.; Braegger, C.P.; Bronsky, J.; et al. Consensus guidelines of ECCO/ESPGHAN on the medical management of pediatric Crohn's disease. J. Crohns. Colitis 2014, 8, 1179-1207. [CrossRef] [PubMed]

3. Zachos, M.; Tondeur, M.; Griffiths, A.M. Enteral nutritional therapy for induction of remission in Crohn's disease. Cochrane Database Syst. Rev. 2007, 4, CD000542. [CrossRef] [PubMed]

4. Borrelli, O.; Cordischi, L.; Cirulli, M.; Paganelli, M.; Labalestra, V.; Uccini, S.; Russo, M.P.; Cucchiara, S. Polymeric Diet Alone Versus Corticosteroids in the Treatment of Active Pediatric Crohn's Disease: A Randomized Controlled Open-Label Trial. Clin. Gastroenterol. Hepatol. 2006, 4, 744-753. [CrossRef]

5. Gerasimidis, K.; Bertz, M.; Hanske, L.; Junick, J.; Biskou, O.; Aguilera, M.; Garrick, V.; Russell, K.R.; Blaut, P.; McGrogan, P.; et al. Decline in Presumptively Protective Gut Bacterial Species and Metabolites Are Paradoxically Associated with Disease Improvement in Pediatric Crohn's Disease During Enteral Nutrition. Inflamm. Bowel Dis. 2014, 20, 861-871. [CrossRef]

6. Buchanan, E.; Gaunt, W.W.; Cardigan, T.; Garrick, V.; McGrogan, P.; Russel, R.K. The use of exclusive enteral nutrition for induction of remission in children with Crohn's disease demonstrates that disease phenotype does not influence clinical remission. Aliment. Pharmacol. Ther. 2009, 30, 501-507. [CrossRef]

7. Critch, J.; Day, A.S.; Otley, A.; King-Moore, C.; Teitelbaum, J.E.; Shashidhar, H. Use of Enteral Nutrition for the Control of Intestinal Inflammation in Pediatric Crohn Disease. J. Pediatr. Gastroenterol. Nutr. 2012, 54, 298-305. [CrossRef] [PubMed]

8. Turner, D.; Levine, A.; Escher, J.C.; Griffiths, A.M.; Russell, R.K.; Dignass, A.; Dias, J.A.; Bronsky, J.; Braegger, C.P.; Cucchiara, S.; et al. Management of Pediatric Ulcerative Colitis. J. Pediatr. Gastroenterol. Nutr. 2012, 55, 340-361. [CrossRef] [PubMed] 
9. Leach, S.T.; Mitchell, H.M.; Eng, W.R.; Zhang, L.; Day, A.S. Sustained modulation of intestinal bacteria by exclusive enteral nutrition used to treat children with Crohn's disease. Aliment. Pharmacol. Ther. 2008, 28, 724-733. [CrossRef]

10. Chen, W.-X. Enteric microbiota leads to new therapeutic strategies for ulcerative colitis. World J. Gastroenterol. 2014, 20, 15657. [CrossRef]

11. Fite, A.; Macfarlane, S.; Furrie, E.; Bahrami, B.; Cummings, H.J.; Steinke, T.D.; Macfarlane, T.G. Longitudinal Analyses of Gut Mucosal Microbiotas in Ulcerative Colitis in Relation to Patient Age and Disease Severity and Duration. J. Clin. Microbiol. 2013, 51, 849-856. [CrossRef] [PubMed]

12. Wedrychowicz, A.; Kowalska-Duplaga, K.; Jedynak-Wasowicz, U.; Pieczarkowski, S.; Sladek, M.; Tomasik, P.; Fyderek, K. Serum Concentrations of VEGF and TGF- $\beta 1$ During Exclusive Enteral Nutrition in IBD. J. Pediatr. Gastroenterol. Nutr. 2011, 53, 150-155. [CrossRef]

13. Turner, D.; Ruemmele, F.M.; Orlanski-Meyer, E.; Griffiths, A.M.; de Carpi, J.M.; Bronsky, J.; Veres, G.; Aloi, M.; Strisciuglio, C.; Braegger, C.P.; et al. Management of Paediatric Ulcerative Colitis, Part 1. J. Pediatr. Gastroenterol. Nutr. 2018, 67, 292-310. [CrossRef] [PubMed]

14. Pigneur, B.; Lepage, P.; Mondot, S.; Schmitz, J.; Goulet, O.; Doré, J.; Ruemmele, F.M. Mucosal Healing and Bacterial Composition in Response to Enteral Nutrition Vs Steroid-based Induction Therapy-A Randomised Prospective Clinical Trial in Children With Crohn's Disease. J. Crohn's Colitis 2018, 13, 846-855. [CrossRef] [PubMed]

15. Quince, C.; Ijaz, U.Z.; Loman, N.; Eren, A.M.; Saulnier, D.; Russell, J.; Haig, S.J.; Calus, S.T.; Quick, J.; Barclay, A.; et al. Extensive Modulation of the Fecal Metagenome in Children with Crohn's Disease During Exclusive Enteral Nutrition. Am. J. Gastroenterol. 2015, 110, 1718-1729. [CrossRef] [PubMed]

16. Svolos, V.; Hansen, R.; Nichols, B.; Quince, C.; Ijaz, U.Z.; Papadopoulou, R.T.; Edwards, C.A.; Watson, D.; Alghamdi, A.; Brejnrod, A.; et al. Treatment of Active Crohn's Disease with an Ordinary Food-based Diet That Replicates Exclusive Enteral Nutrition. Gastroenterology 2019, 156, 1354-1367. [CrossRef]

17. Turner, D.; Otley, A.R.; Mack, D.; Hyams, J.; de Bruijne, J.; Uusoue, K.; Walters, T.D.; Zachos, M.; Mamula, P.; Beaton, D.E.; et al. Development, Validation, and Evaluation of a Pediatric Ulcerative Colitis Activity Index: A Prospective Multicenter Study. Gastroenterology 2007, 133, 423-432. [CrossRef] [PubMed]

18. Hyams, J.S.; Ferry, G.D.; Mandel, F.S.; Gryboski, J.D.; Kibort, P.M.; Kirschner, B.S.; Griffiths, A.M.; Katz, A.J.; Grand, R.J.; Boyle, J.T.; et al. Development and validation of a pediatric Crohn's disease activity index. J. Pediatr. Gastroenterol. Nutr. 1991, 12, 439-447. Available online: http://www.ncbi.nlm.nih.gov/pubmed/ 1678008 (accessed on 1 January 2016). [CrossRef]

19. Whelan, F.J.; Verschoor, C.P.; Stearns, J.C.; Rossi, L.; Luinstra, K.; Loeb, M.; Smieja, M.; Johnstone, J.; Surette, M.G.; Bowdish, D.M.E. The Loss of Topography in the Microbial Communities of the Upper Respiratory Tract in the Elderly. Ann. Am. Thorac. Soc. 2014, 11, 513-521. [CrossRef]

20. Bartram, A.K.; Lynch, M.D.J.; Stearns, J.C.; Moreno-Hagelsieb, G.; Neufeld, J.D. Generation of Multimillion-Sequence 16S rRNA Gene Libraries from Complex Microbial Communities by Assembling Paired-End Illumina Reads. Appl. Environ. Microbiol. 2011, 77, 3846-3852. [CrossRef]

21. Cleynen, I.; Boucher, G.; Jostins, L.; Schumm, L.P.; Zeissig, S.; Ahmad, T.; Andersen, V.; Andrews, J.M.; Annese, V.; Brand, S.; et al. Inherited determinants of Crohn's disease and ulcerative colitis phenotypes: A genetic association study. Lancet 2016, 387, 156-167. [CrossRef]

22. Aschard, H.; Laville, V.; Tchetgen, E.T.; Knights, D.; Imhann, F.; Seksik, P.; Zaitlen, N.; Silverberg, M.S.; Cosnes, J.; Weersma, R.K.; et al. Genetic effects on the commensal microbiota in inflammatory bowel disease patients. PLoS Genet. 2019, 15, e1008018. [CrossRef] [PubMed]

23. Knights, D.; Lassen, K.G.; Xavier, R.J. Advances in inflammatory bowel disease pathogenesis: Linking host genetics and the microbiome. Gut 2013, 62, 1505-1510. [CrossRef] [PubMed]

24. Schwerd, T.; Frivolt, K.; Clavel, T.; Lagkouvardos, I.; Katona, G.; Mayr, D.; Uhlig, H.H.; Haller, D.; Koletzko, S.; Bufler, P. Exclusive enteral nutrition in active pediatric Crohn disease: Effects on intestinal microbiota and immune regulation. J. Allergy Clin. Immunol. 2016, 138, 592-596. [CrossRef] [PubMed]

25. D’Argenio, V.; Precone, V.; Casaburi, G.; Miele, E.; Martinelli, M.; Staiano, A.; Salvatore, F.; Sacchetti, L. An Altered Gut Microbiome Profile in a Child Affected by Crohn's Disease Normalized After Nutritional Therapy. Am. J. Gastroenterol. 2013, 108, 851-852. [CrossRef] 
26. Kaakoush, N.O.; Day, A.S.; Leach, S.T.; Lemberg, D.A.; Nielsen, S.; Mitchell, H.M. Effect of Exclusive Enteral Nutrition on the Microbiota of Children with Newly Diagnosed Crohn's Disease. Clin. Transl. Gastroenterol. 2015, 6, e71. [CrossRef]

27. Yu, T.; Yu, Q.; Chen, X.; Zhou, L.; Wang, Y.; Yu, C. Exclusive enteral nutrition protects against inflammatory bowel disease by inhibiting NF-кB activation through regulation of the p38/MSK1 pathway. Int. J. Mol. Med. 2018, 42, 1305-1316. [CrossRef]

(C) 2020 by the authors. Licensee MDPI, Basel, Switzerland. This article is an open access article distributed under the terms and conditions of the Creative Commons Attribution (CC BY) license (http://creativecommons.org/licenses/by/4.0/). 